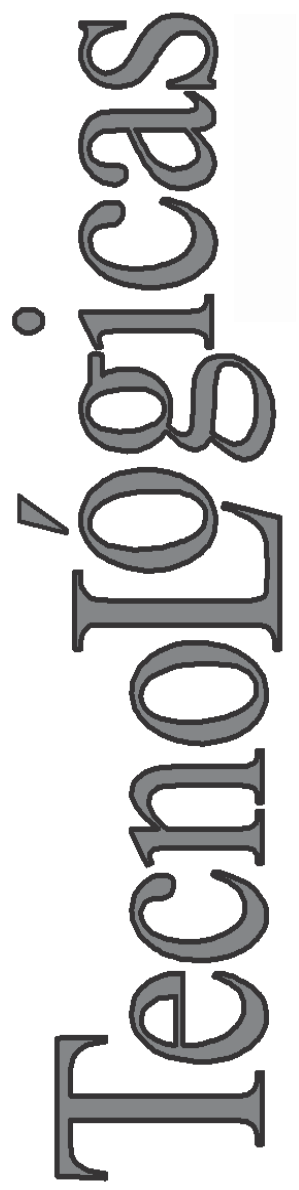

\title{
Plataforma de Modelado y Simulación de Sistemas de Generación Fotovoltaicos
}

\section{Platform for Modeling and Simulation of Photovoltaic Generation Systems}

\author{
Anny A. Arroyave-Berrio ${ }^{1}$ \\ Manuel M. Goez-Mora ${ }^{2}$ \\ Eliana I. Arango-Zuluaga ${ }^{3}$ \\ Carlos A. Ramos-Paja ${ }^{4}$ \\ Sergio I. Serna-Garcés ${ }^{5}$
}

1 Facultad de Minas, Departamento de Energía Eléctrica y Automática, Universidad Nacional de

Colombia Sede Medellín, Medellín-Colombia aaaroyaveb@unal.edu.co

2 Facultad de Minas, Departamento de Energía Eléctrica y Automática, Universidad Nacional de

Colombia Sede Medellín, Medellín-Colombia mmgoezm@unal.edu.co

3 Facultad de Minas, Departamento de Energía Eléctrica y Automática, Universidad Nacional de Colombia Sede Medellín, Medellín-Colombia eiarangoz@unal.edu.co

4 Facultad de Minas, Departamento de Energía Eléctrica y Automática, Universidad Nacional de

Colombia Sede Medellín, Medellín-Colombia caramosp@unal.edu.co

5 Departamento de Electrónica y Telecomunicaciones, Instituto Tecnológico Metropolitano, Medellín-Colombia sergioserna@itm.edu.co 


\section{Resumen}

Se presenta el desarrollo de una plataforma de modelado y simulación usando Matlab. La plataforma tiene cuatro modelos de paneles fotovoltaicos predeterminados. Identifica los parámetros de cada uno, para un panel fotovoltaico dado, con base en datos experimentales de voltaje, corriente y condiciones ambientales. También genera cuatro bloques de simulación en Matlab-Simulink y Psim que modelan el panel seleccionado. Para la validación de la plataforma se utilizaron los paneles disponibles en el Instituto Tecnológico Metropolitano (ITM).

\section{Palabras clave}

Paneles fotovoltaicos; caracterización de modelos; función Lambert W; algoritmo de Levenberg-Mardquardt.

\section{Abstract}

A platform for modeling and simulation using Matlab is presented. The platform has four models of photovoltaic panels. It identifies the parameters of each one, for a given solar panel, based on experimental data of voltage, current and environmental conditions. Also the platform generates four blocks, for using in Matlab-Simulink and Psim simulation tools. The experimental validation of the platform was made using the PV panels of the Metropolitan Technological Institute (ITM) Lab.

\section{Keywords}

Photovoltaic PV panels; characterization of models; Lambert W function; Levenberg-Mardquardt algorithm. 


\section{INTRODUCCIÓN}

La extracción de energía solar usando sistemas fotovoltaicos es uno de los tópicos de investigación más activos en ingeniería, ya que ofrecen la posibilidad de generar energía eléctrica en el sitio requerido, así como proveerla a regiones poco accesibles por redes convencionales (Petrone et al., 2007). Este tipo de energía renovable presenta algunos inconvenientes ya que en casos donde la irradiación solar, temperatura ambiente y perfiles de carga cambian rápidamente, es difícil extraer la máxima potencia disponible debido a efectos de sombreado y falla en la predicción del punto óptimo de operación (Petrone \& Ramos-Paja, 2011). Por esto se requieren estrategias de optimización que incrementen la viabilidad económica y la producción energética de las celdas solares (Ramos-Paja et al, 2012). Para llevar a cabo esta optimización se hacen necesarias herramientas de modelado, análisis y simulación de sistemas de generación fotovoltaicos enmarcados en las condiciones geográficas-climáticas y de perfiles de carga de nuestro país. Tener un modelo del comportamiento de un panel fotovoltaico ayuda a diseñar convertidores que persigan siempre el punto de potencia máxima o emuladores que imiten el comportamiento de un panel solar, lo cual es muy útil en el caso de comprobar nuevos algoritmos de control.

El objetivo de este trabajo es crear una GUI en MATLAB que automatice la caracterización de un panel solar. Para esto se utilizaron los modelos de paneles mencionados en (Petrone \& RamosPaja, 2011; Gow \& Manning, 1999; Petrone et al., 2007). El primer modelo tratado es un modelo linealizado alrededor del punto de máxima potencia, este modelo es el más impreciso en cuanto al seguimiento del comportamiento del panel solar, pero presenta un ajuste satisfactorio en el punto de máxima potencia, que es el más usado en el diseño de los convertidores. Sin embargo, en el caso de los emuladores es necesario un modelo que imite punto a punto el comportamiento del panel, con este fin se estudiaron los tres modelos restantes, entre estos se encuentran el modelo de la exponencial trabajado en Gow y Manning (1999), el del diodo bypass usado en Petrone et al. (2007) y el modelo sin resistencia serie estudiado en Petrone y Ramos-Paja (2011). Las ecuaciones de 
estos tres modelos, presentan una estructura matemática compleja no lineal, siendo el modelo sin resistencia serie el más sencillo ya que tiene una ecuación explícita, propiedad con la que no cuentan los dos modelos restantes. En el caso de las ecuaciones implícitas se acude a la función Lambert como se presenta en García (2006). Esta función tiene un alto costo computacional que es recompensado con una mejor precisión en el modelo. Una vez explícita la función, el paso a seguir es hallar los parámetros del modelo, para esto actualmente se emplean técnicas alternativas como los algoritmos genéticos (Hong \& Weixiang, 2008), las redes neuronales (Mellit et al., 2003), y la evolución diferencial (da Costa et al., 2010), entre otros. En este trabajo se utiliza el algoritmo de Levenberg-Mardquardt propuesto en (Gow \& Manning, 1999).

El artículo está organizado como sigue: En la sección 2 se presenta el desarrollo de la plataforma de caracterización, describiendo detalladamente cada modelo utilizado, el algoritmo y la generación de los bloques de simulación. A continuación, en la sección 3 se muestran los resultados de la validación experimental, y finalmente las conclusiones del trabajo realizado y los futuros desarrollos se plantean en la sección 4 .

\section{DESARROLlo DE LA PLATAforma DE MODElado $Y$ SIMULACIÓN}

La plataforma de caracterización consta de dos partes, la primera de ellas es una GUI de Matlab, que obtiene los parámetros de un modelo de panel fotovoltaico para un panel seleccionado, a partir de datos experimentales de voltaje, corriente y condiciones ambientales (temperatura e irradiación), que deben ser ingresados por el usuario. En la segunda parte de la plataforma, se generan cuatro bloques de simulación en Simulink-Matlab y Psim para el panel seleccionado, cada bloque se corresponde con cada uno de los modelos con los parámetros ya calculados. Estos bloques facilitan al usuario el planteamiento de simulaciones con los modelos calculados.

La plataforma tiene cuatro modelos predeterminados y tres opciones; la primera opción es de validación del modelo con los 
parámetros hallados, la segunda es la simulación de cambios en la irradiación y la tercera es la simulación de cambios en la temperatura. Los resultados se muestran en dos planos cartesianos, el primero grafica la curva corriente vs voltaje y el segundo la curva potencia vs voltaje. Como se muestra en la Fig. 1.

\subsection{Modelos Predeterminados}

A continuación se describe la nomenclatura utilizada en las ecuaciones para cada modelo:

$\boldsymbol{V}=$ Voltaje entregado por el panel; $\boldsymbol{I}=$ Corriente entregada por el panel; $\boldsymbol{I}_{\boldsymbol{P H}}=$ Corriente de corto circuito; $\boldsymbol{I}_{\boldsymbol{S}}=$ Corriente de saturación del diodo; $\boldsymbol{I}_{\boldsymbol{S} \boldsymbol{d} \boldsymbol{b}}=$ Corriente de saturación del diodo bypass; $\boldsymbol{A}=$ Factor del diodo; $\boldsymbol{R}_{\boldsymbol{S}}=$ Resistencia serie del modelo; $\boldsymbol{R}_{\boldsymbol{P}}=$ Resistencia paralelo del modelo; $\boldsymbol{e}=$ Carga del electrón, $1.6 e^{-19} C ; \boldsymbol{K}=$ Constante de Boltzmann's, $1.38 e^{-23} ; \boldsymbol{T}=$ Temperatura ambiente, grados Kelvin.
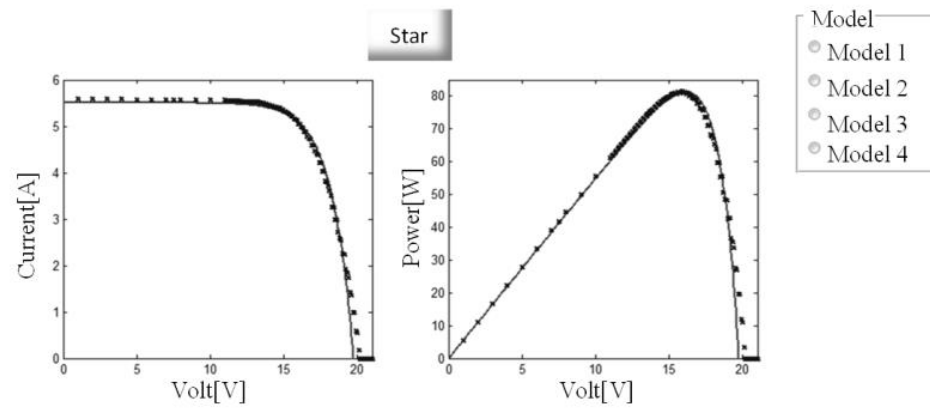

Fig. 1. Diseño de la plataforma

\subsubsection{Modelo 1}

El modelo de la exponencial trabajado en Gow y Manning (1999), es regido por (1) y su circuito representativo se muestra en la Fig. 2. Este modelo es ideal para ser implementado en análisis donde el tiempo de simulación es irrelevante, ya que el costo computacional para la solución de (1) es muy alto y exige un tiempo de cálculo mayor que en otros modelos. 


$$
I=I_{P H}-\left[e^{\frac{e\left(V+I R_{S}\right)}{A K T}}-1\right] I_{S}-\frac{V+I R_{S}}{R_{P}}
$$

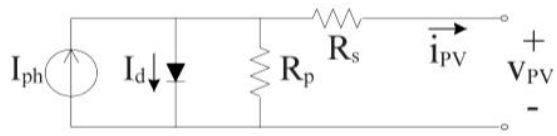

Fig. 2. Circuito representativo del modelo 1

\subsubsection{Modelo 2}

El modelo lineal es representativo sólo alrededor del punto de máxima potencia, pero es el más sencillo, por lo tanto es uno de los más utilizados para diseño de convertidores, controladores de convertidores y análisis de seguimientos del punto de máxima potencia (MPPT). El comportamiento de este modelo se puede expresar por medio de (2) y su circuito representativo se muestra en la Fig. 3.

$$
I=I_{P H}-\frac{V}{R_{P}}
$$

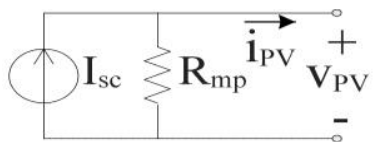

Fig. 3. Circuito representativo del modelo 2

\subsubsection{Modelo 3}

El modelo sin resistencia $R s$ estudiado en Petrone y RamosPaja (2011), es regido por (3) y se representa por medio del circuito de la Fig. 4. Este modelo ofrece una representación satisfactoria del comportamiento del panel en toda su trayectoria y es explícito, lo que se considera una ventaja en comparación con el modelo 1 ya que los cálculos para la estimación de parámetros requieren de un menor esfuerzo computacional.

$$
I=I_{P H}-\left[e \frac{e V}{A K T}-1\right] I_{S}-\frac{V}{R_{P}}
$$




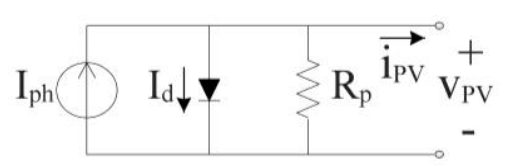

Fig. 4. Circuito representativo del modelo 3

\subsubsection{Modelo 4}

El modelo del diodo bypass usado en Petrone et al. (2007), permite una representación realista del panel por la inclusión del diodo de bypass, que facilita el estudio del desgaste del panel causado por las pequeñas corrientes que transitan por él cuando hay interconexión de paneles. Es regido por (4) y se representa por medio del circuito mostrado en la Fig. 5.

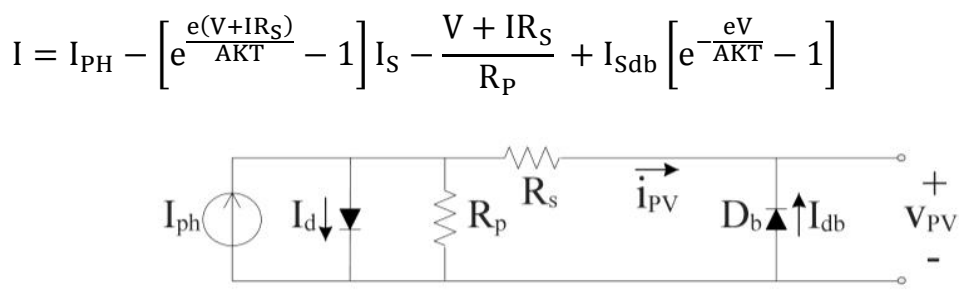

Fig. 5. Circuito representativo del modelo 4

\subsection{Algoritmo utilizado para la Convergencia de los Modelos}

Como se mencionó anteriormente, la plataforma utiliza la función de Lambert para el cálculo de la corriente $I$ en los modelos 1 y 4 , ya que al hacer parte de una exponencial, no es posible hallar los parámetros por medio del uso de métodos tradicionales.

Posterior a esto, se utiliza el algoritmo de LevenbergMarquard para hallar los parámetros de las curvas que modelan el panel fotovoltaico. Este algoritmo es eficiente en cuanto al ajuste de la curva, sin embargo presenta un inconveniente referente a la asignación de los valores iniciales. Este inconveniente se debe a que el algoritmo encuentra mínimos de error locales, entonces si el valor inicial del parámetro no está cerca del valor real, el algoritmo podría no converger al punto deseado. Por esta razón es importante definir los valores iniciales según el tipo panel con el cual se esté trabajando, estos valores se pueden obtener de la hoja de datos (datasheet) del panel, de bases de datos en la web o de pa- 
rámetros previos. La Fig. 6 esboza el algoritmo utilizado para la convergencia de los modelos.

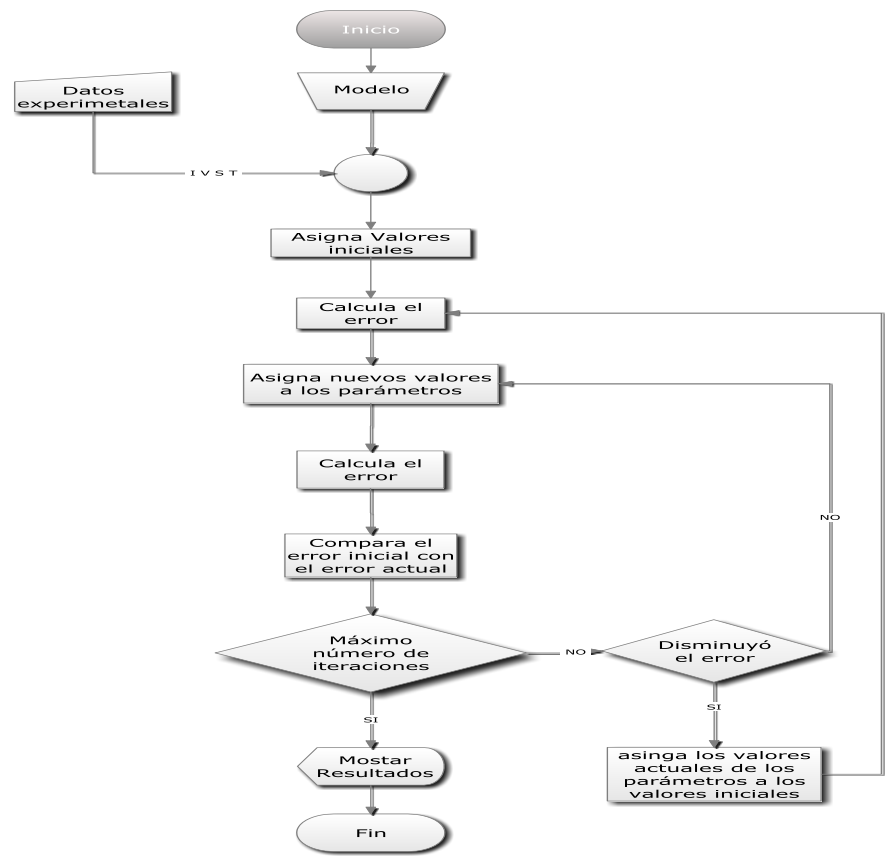

Fig. 6. Diagrama de Flujo

\subsection{Generación de Bloques en Matlab y Psim}

La generación del bloque para su utilización en MatlabSimulink se realizó por medio del bloque Embedded MATLAB Function y el código de la función en Matlab representa el modelo escogido con los respectivos parámetros arrojados por la plataforma. El bloque, que se puede observar en la Fig. 7, tiene tres entradas (Voltaje - Irradianza - Temperatura) y dos salidas (Corriente Potencia), con el fin de simular el comportamiento de las variables de interés de los paneles caracterizados

Para los bloques en Psim se ingresaron los modelos de circuitos en los cuales la irradiación y la temperatura son las variables que controlan una fuente de corriente dependiente $(I p h)$. El diodo es 
representado por un elemento no lineal $i=F(v, x)$ y la carga es simulada por medio de una fuente de voltaje (Fig. 8). Se generaron los bloques para cada uno de los modelos después de hallar sus parámetros usando la plataforma, por cuestiones de espacio, sólo se muestran los bloques del modelo 3 como un ejemplo.

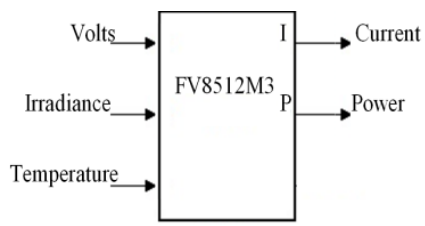

Fig. 7. Bloque para uso en simulaciones con Matlab-Simulink

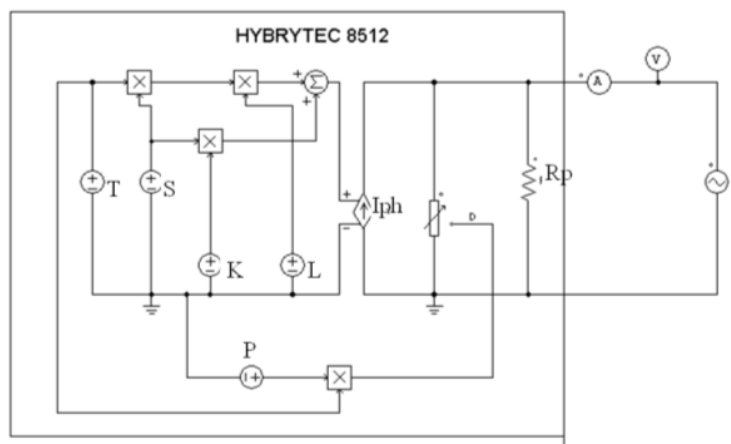

Fig. 8. Bloque para uso en simulaciones con PSIM

\section{VALIDACIÓN EXPERIMENTAL}

La toma de datos fue realizada en el Laboratorio de fuentes de energía renovable del ITM, con tres paneles fotovoltaicos Hybrytec 8512. Para llevar a cabo esta prueba se necesitó la carga electrónica DC programable 8510, un computador con el software PV8500, que es el software de que controla la carga y un sensor de irradiación y temperatura.

En la Fig. 9 se observa que la mayor densidad de datos se está tomando alrededor el punto de máxima potencia, debido a que este es el que requiere una mejor aproximación en el modelo y después de la realización de varias pruebas en las tomas de datos se deci- 
dió aumentar la densidad de datos en esta zona para obtener mejores resultados.

Para la validación se superpusieron las respuestas obtenidas en cada uno de los cuatro modelos, después de hallados sus parámetros con la plataforma GUI de modelado y simulación (línea continua en las gráficas), con los datos experimentales obtenidos al usar los paneles fotovoltaicos Hybrytec 8512 (puntos en las gráficas). Los resultados se muestran en la Fig. 9 respectivamente para cada modelo. La precisión se evaluó por medio de (5) que representa el error cuadrático medio del ajuste. Donde $n$ es el número de datos tomados.

$\mathrm{E}_{\mathrm{cm}}=\sqrt{\frac{\sum_{\mathrm{k}=1}^{\mathrm{n}}(\mathrm{I}(\mathrm{k})-\operatorname{Iestimada}(\mathrm{k}))^{2}}{\mathrm{n}}}$

Debido a que el modelo 2 es una linealización alrededor del punto de máxima potencia, el error $E c m$ fue calculado con respecto a este punto y los puntos más cercanos. Según el cálculo del Ecm, se aprecia la mejor aproximación y un comportamiento similar en los modelos 1 y 4, ya que la única diferencia entre estos es el diodo de bypass ideal para la interconexión de paneles fotovoltaicos, que no entra en funcionamiento si sólo se está simulando un panel.

Es importante aclarar que el modelo 2 solo es representativo en un rango de $\pm 1 \mathrm{~V}$ alrededor del punto de máxima potencia, los demás modelos son modelos representativos para todo el intervalo de datos tomados experimentalmente. En general se observa que los modelos calculados por la plataforma de modelado, realizan una buena aproximación del comportamiento de los paneles fotovoltaicos. Los modelos 1 y 4 presentan una mejor aproximación antes del punto de máxima potencia y el modelo 3, después del punto de máxima potencia.

Para la obtención de las gráficas teóricas de cada modelo (línea continua), se utilizaron directamente los resultados entregados por la GUI. Es importante anotar que las mismas gráficas se pueden obtener en Matlab-Simulink o en Psim, utilizando para la simulación los bloques programados en estas plataformas. Estas gráficas no se demuestran aquí por cuestiones de espacio 


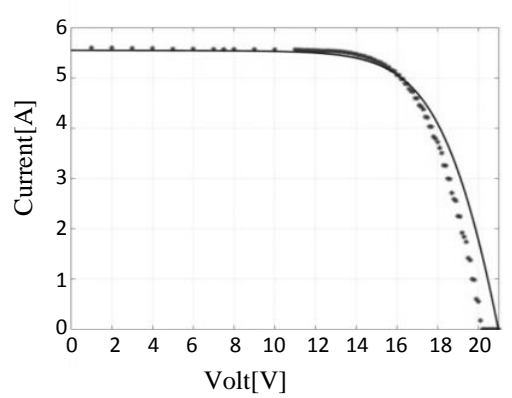

a)

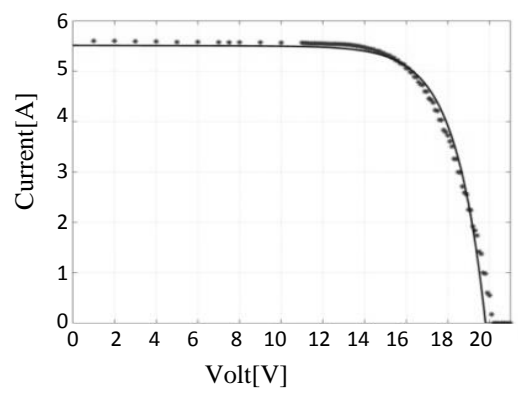

c)

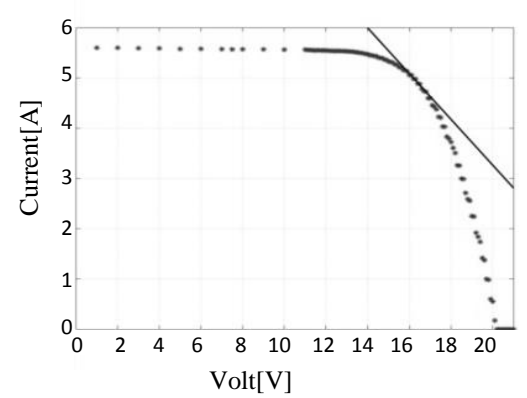

b)

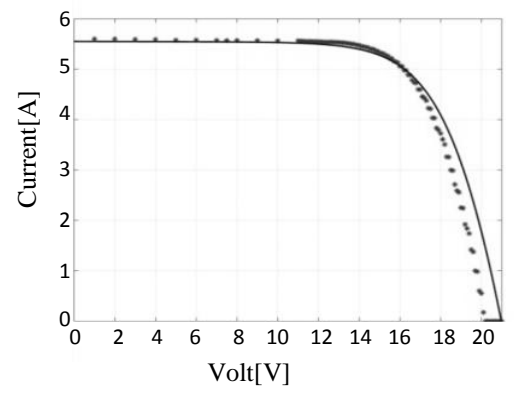

d)

Fig. 9. Validación de los datos con a) el modelo $1 . E c m=0.0253, b)$ el modelo 2. $E c m=0.03$, c) el modelo $3 . E c m=0.0437$, y d) el modelo $4 . E c m=0.0256$

\section{CONCLUSIONES}

Los resultados obtenidos demuestran que la plataforma calcula los parámetros y simula de forma correcta el comportamiento de un panel fotovoltaico permitiendo obtener curvas estáticas estimadas frente a cambios ambientales. Además los bloques generados en Matlab-Simulink y Psim replican de forma correcta la respuesta del modelo obtenido por la plataforma.

De los diversos modelos estudiados, se concluye que el modelo sin la resistencia en serie es un modelo óptimo para el proceso de simulación y emulación de paneles fotovoltaicos, ya que permite simplificar cálculos y obtener un ajuste de curva con un error no mayor al 4\% del error obtenido con modelo exponencial, el cual es 
más preciso, pero requiere del uso de la función Lambert $\mathrm{W}$, la cual demanda un gran costo computacional. Por tanto, para tareas de emulación en tiempo real, los modelos que presenten el uso de esta función requieren equipos de alta eficiencia computacional lo cual dificulta su implementación. Para estos casos es recomendable utilizar otros recursos como por ejemplo sistemas expertos.

Se comprueba que el efecto del diodo bypass es despreciable cuando no existe conexión entre paneles y debe ser tenido en cuenta en el modelo del panel cuando haya interconexión. Para realizar la toma de datos, es recomendable contar con un ambiente artificial donde la irradiación y la temperatura sean controlables, esto con el fin de evitar cambios inesperados de irradiación que afecten al panel y que el sensor no detecte; el ejemplo más claro que ilustra esta situación es el efecto sombra.

\section{AGRADECIMIENTOS}

Este trabajo ha sido desarrollado en la línea de investigación en Modelado y control de fuentes de potencia eléctrica del Grupo de Automática de la Universidad Nacional - GAUNAL, bajo los proyectos "MPPT Vectorial" y "Smart-Alen", en colaboración con el grupo de Automática y Electrónica del Instituto Tecnológico Metropolitano ITM.

\section{REFERENCIAS}

da Costa, W.T., Fardin, J.F., Simonetti, D.S.L. \& Neto, L. (2010). Identification of photovoltaic model parameters by Differential Evolution. In IEEE ICIT International Conference on Industrial Technology. 931936.

García, F. J., Ortiz-Conde, A., Malobabic, S. (2006). Applications of Lambert's Function in Electronics. Rev. Universidad, Ciencia y Tecnología, Vol. 10, (40).

Gow, J.A. \& Manning, C.D. (1999). Development of a photovoltaic array model for use in power-electronics simulation studies. Rev. IEEE Proceedings on Electric Power Applications. Vol.146. No.2. 193-200.

Hong, T.Z. \& Weixiang, S. (2008). Application of genetic algorithms in the design of a solar array-exclusive standalone photovoltaic system. In 
IEEE PECON 2008, 2nd International Power and Energy Conference. 451-456.

Mellit, A., Benghanem, M., Hadj A. A. \& Guessoum, A. (2003). Modelling of sizing the photovoltaic system parameters using artificial neural network. In Proceedings of CCA 2003 IEEE Conference on Control Applications. Vol.1. 353- 357.

Petrone, G., Spagnuolo, G., \& Vitelli, M. (2011). A multivariable perturband-observe maximum power point tracking technique applied to a single-stage photovoltaic inverter. Industrial Electronics, IEEE Transactions on, 58(1), 76-84.

Petrone, G., \& Ramos-Paja, C. A. (2011). Modeling of photovoltaic fields in mismatched conditions for energy yield evaluations. Electric Power Systems Research, 81(4), 1003-1013.

Petrone, G., Spagnuolo, G., \& Vitelli, M. (2007). Analytical model of mismatched photovoltaic fields by means of Lambert W-function. Solar Energy Materials and Solar Cells, 91(18), 1652-1657.

Ramos-Paja, C. A., Giral, R., \& Arango Zuluaga, E. I. (2012). Distributed maximum power point tracking in photovoltaic applications: active bypass DC/DC converter. Revista Facultad de Ingeniería Universidad de Antioquia, (64), 32-44. 